\title{
Spectral analysis of the hypoelliptic Robin problem
}

\author{
Kazuaki Taira \\ Dedicated to the memory of Professor Kyuya Masuda (1937-2018)
}

Received: date / Accepted: date

\begin{abstract}
This paper is devoted to a functional analytic approach to the study of the hypoelliptic Robin problem for a second-order, uniformly elliptic differential operator with a complex parameter $\lambda$, under the probabilistic condition that either the absorption phenomenon or the reflection phenomenon occurs at each point of the boundary. We solve the long-standing open problem of the asymptotic eigenvalue distribution for the homogeneous Robin problem when $|\lambda|$ tends to $\infty$. More precisely, we prove the spectral properties of the closed realization of the uniformly elliptic differential operator, similar to the elliptic (non-degenerate) case. However, in the degenerate case we cannot use Green's formula to characterize the adjoint operator of the closed realization. Hence, we shift our attention to its resolvent. In the proof, we make use of the Boutet de Monvel calculus in order to study the resolvent and its adjoint in the framework of $L^{2}$ Sobolev spaces.
\end{abstract}

Keywords Robin problem, hypoelliptic operator, non self-adjoint eigenvalue problem, asymptotic eigenvalue distribution, Boutet de Monvel calculus

Mathematics Subject Classification (2010) Primary 35J25; Secondary 35S05, 47D03, 35P20

\section{Introduction}

Let $\Omega$ be a bounded domain of Euclidean space $\mathbf{R}^{n}, n \geq 2$, with smooth boundary $\Gamma=\partial \Omega$; its closure $\bar{\Omega}=\Omega \cup \Gamma$ is an $n$-dimensional, compact smooth manifold with boundary $\Gamma$. Without loss of generality, we may assume that the domain $\Omega$ is a relatively compact open subset of an $n$-dimensional, compact smooth manifold $M$ without boundary. The manifold $M$ is called the double of $\Omega$ (see [19]).

We let

$$
A u=\sum_{i, j=1}^{n} a^{i j}(x) \frac{\partial^{2} u}{\partial x_{i} \partial x_{j}}+\sum_{i=1}^{n} b^{i}(x) \frac{\partial u}{\partial x_{i}}+c(x) u
$$

Kazuaki Taira

Institute of Mathematics, University of Tsukuba, Tsukuba 305-8571, Japan

E-mail: taira@math.tsukuba.ac.jp 
be a second-order, uniformly elliptic differential operator defined on the double $M$ with real coefficients such that:

(1) The $a^{i j}(x)$ are the components of a $C^{\infty}$ symmetric contravariant tensor of type $\left(\begin{array}{l}2 \\ 0\end{array}\right)$ on $M$ and there exists a constant $a_{0}>0$ such that

$$
\sum_{i, j=1}^{n} a^{i j}(x) \xi_{i} \xi_{j} \geq a_{0}|\xi|^{2} \quad \text { on } T^{*}(M),
$$

where $T^{*}(M)$ is the cotangent bundle of $M$.

(2) $b^{i} \in C^{\infty}(M)$ for $1 \leq i \leq n$.

(3) $c \in C^{\infty}(M)$ and $c(x) \leq 0$ in $\Omega$.

In this paper, we consider the following Robin boundary condition

$$
B \gamma u=a\left(x^{\prime}\right) \frac{\partial u}{\partial \boldsymbol{\nu}}+\left.b\left(x^{\prime}\right) u\right|_{\Gamma} .
$$

Here:

(4) $a\left(x^{\prime}\right)$ and $b\left(x^{\prime}\right)$ are real-valued, smooth functions on the boundary $\Gamma$.

(5) $\partial / \partial \boldsymbol{\nu}$ is the conormal derivative associated with the operator $A$ :

$$
\frac{\partial}{\partial \boldsymbol{\nu}}=\sum_{i=1}^{n} a^{i j}\left(x^{\prime}\right) n_{j} \frac{\partial}{\partial x_{i}},
$$

where $\mathbf{n}=\left(n_{1}, n_{2}, \ldots, n_{n}\right)$ is the unit outward normal to the boundary $\Gamma$.

The purpose of this paper is to study the following homogeneous Robin problem with a complex parameter $\lambda$ : Given a function $f(x)$ defined in $\Omega$, find a function $u(x)$ in $\Omega$ such that

$$
\begin{cases}(A-\lambda) u=f & \text { in } \Omega, \\ B \gamma u=a\left(x^{\prime}\right) \frac{\partial u}{\partial \boldsymbol{\nu}}+\left.b\left(x^{\prime}\right) u\right|_{\Gamma}=0 . & \text { on } \Gamma .\end{cases}
$$

In this paper, we impose the following two conditions on the functions $a\left(x^{\prime}\right)$ and $b\left(x^{\prime}\right):$ :

(H.1) $a\left(x^{\prime}\right) \geq 0$ and $b\left(x^{\prime}\right) \geq 0$ on $\Gamma$.

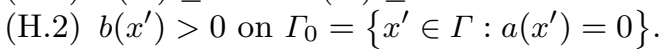

The intuitive meaning of conditions (H.1) and (H.2) is that the absorption phenomenon occurs at each point of the set $\Gamma_{0}$, while the reflection phenomenon occurs at each point of the set $\Gamma \backslash \Gamma_{0}=\left\{x^{\prime} \in \Gamma: a\left(x^{\prime}\right)>0\right\}$.

We give a simple example of conditions (H.1) and (H.2) in the unit disk in the plane $\mathbf{R}^{2}$ :

Example 1.1 Let $\Omega=\left\{\left(x_{1}, x_{2}\right) \in \mathbf{R}^{2}: x_{1}^{2}+x_{2}^{2}<1\right\}$ be the disk with the boundary $\Gamma=\left\{\left(x_{1}, x_{2}\right) \in \mathbf{R}^{2}: x_{1}^{2}+x_{2}^{2}=1\right\}$. For a local coordinate system $x_{1}=\cos \theta$ and $x_{2}=\sin \theta$ with $\theta \in[0,2 \pi]$ on the circle $\Gamma$, we define a function $a\left(x_{1}, x_{2}\right)$ by the formula

$$
a\left(x_{1}, x_{2}\right)=a(\cos \theta, \sin \theta)
$$




$$
= \begin{cases}e^{\frac{2}{\pi}-\frac{1}{\theta}}\left(1-e^{\frac{2}{\pi}+\frac{1}{\theta-\frac{\pi}{2}}}\right) & \text { for } \theta \in\left[0, \frac{\pi}{2}\right], \\ 1 & \text { for } \theta \in\left[\frac{\pi}{2}, \pi\right], \\ e^{\frac{2}{\pi}+\frac{1}{\theta-\frac{3 \pi}{2}}}\left(1-e^{\frac{2}{\pi}-\frac{1}{\theta-\pi}}\right) & \text { for } \theta \in\left[\pi, \frac{3 \pi}{2}\right], \\ 0 & \text { for } \theta \in\left[\frac{3 \pi}{2}, 2 \pi\right],\end{cases}
$$

and let

$$
b\left(x_{1}, x_{2}\right)=1-a\left(x_{1}, x_{2}\right) \quad \text { on } \Gamma .
$$

It is easy to see that the boundary condition $B$ is non-degenerate (or coercive) if and only if either $a\left(x^{\prime}\right)>0$ on $\Gamma$ (the regular Robin case) or $a\left(x^{\prime}\right) \equiv 0$ and $b\left(x^{\prime}\right)>0$ on $\Gamma$ (the Dirichlet case). However, our boundary condition $B$ is degenerate in the Lopatinski-Shapiro sense (see [5, Chapitre V, condition (4.5)]; [12, Chapter XX, Definition 20.1.1]; [21, Chapter 3, p. 194, Definition 1], [31, Chapter II, Condition 11.1])). This is due to the fact that the so-called Lopatinski-Shapiro complementary condition is violated at the points $x^{\prime}$ of $\Gamma_{0}$ ([28, Section 6.6]).

More precisely, if we reduce the study of the problem (1.3) to that of a first order, classical pseudo-differential operator $T$ on the boundary $\Gamma$, then the operator $T$ is of the form

$$
T=a\left(x^{\prime}\right) \sqrt{-\Delta^{\prime}}+b\left(x^{\prime}\right)
$$

where $\Delta^{\prime}$ is the Laplace-Beltrami operator on $\Gamma$ (see [28, Chapter 7]). We can prove that if conditions (H.1) and (H.2) are satisfied, then the operator $T$ has a parametrix $S$ in the Hörmander class $L_{1,1 / 2}^{0}(\Gamma)$ (see the proof of Lemma 6.1). Hence the operator $T$ is hypoelliptic with loss of one derivative on $\Gamma$. Therefore, our Robin problem (1.3) is hypoelliptic in this sense provided conditions (H.1) and (H.2) are satisfied.

\section{Statement of main results}

This paper is devoted to a functional analytic approach to the study of the hypoelliptic Robin problem (1.3) when $|\lambda|$ tends to $\infty$.

2.1 The asymptotic eigenvalue distribution for the Robin problem (1.3)

Our starting point is to state an existence and uniqueness theorem of the problem (1.3) in the framework of $L^{2}$ Sobolev spaces when $|\lambda|$ tends to $\infty$, due to [28, Part (i) of Theorem 1.2] with $p:=2$ :

Theorem 2.1 Assume that conditions (H.1) and (H.2) are satisfied. Then, for every $\theta \in(-\pi, \pi)$ there exists a constant $R(\theta)>0$ continuously depending on $\theta$ such that if $\lambda=r^{2} e^{i \theta}$ satisfies the condition $|\lambda|=r^{2} \geq R(\theta)$, the homogeneous Robin problem (1.3) has a unique solution $u \in H^{2}(\Omega)$ for any $f \in L^{2}(\Omega)$. Moreover, we have the a priori estimate

$$
\|u\|_{H^{2}(\Omega)}+|\lambda|\|u\|_{L^{2}(\Omega)} \leq C(\theta)\|f\|_{L^{2}(\Omega)},
$$

with a constant $C(\theta)>0$ continuously depending on $\theta$.

Here and in the following $H^{s}(\Omega)=H^{s, 2}(\Omega)$ denotes the $L^{2}$ Sobolev space of order $s$ on $\Omega$. 
This rather surprising estimate (2.1) (the elliptic estimate for a degenerate problem) works, since we are considering the homogeneous boundary condition. More precisely, the reader might refer to [28, Section 1.2, Theorem 1.1] for the non-homogeneous case of the hypoelliptic Robin problem (1.3).

Associated with the homogeneous Robin problem (1.3), we introduce a densely defined, closed linear operator

$$
\mathfrak{A}: L^{2}(\Omega) \longrightarrow L^{2}(\Omega)
$$

in the space $L^{2}(\Omega)$ as follows:

(a) The domain $\mathcal{D}(\mathfrak{A})$ of definition is the space

$$
\mathcal{D}(\mathfrak{A})=\left\{u \in H^{2}(\Omega): B \gamma u=0 \text { on } \Gamma\right\} .
$$

(b) $\mathfrak{A} u=A u$ for every $u \in \mathcal{D}\left(\mathfrak{A}_{2}\right)$.

The main purpose of this paper is to prove the following spectral theorem for the closed realization $\mathfrak{A}$ of $A$ in $L^{2}(\Omega)$ (cf. the proof of [29, Theorem 2.3]):

Theorem 2.2 If conditions (H.1) and (H.2) are satisfied, then the closed realization $\mathfrak{A}$ of $A$ enjoys the following five spectral properties:

(i) The spectrum of $\mathfrak{A}$ is discrete and the eigenvalues $\lambda_{j}$ of $\mathfrak{A}$ have finite multiplicities.

(ii) All rays $\arg \lambda=\theta$ different from the negative axis are rays of minimal growth of the resolvent $(\mathfrak{A}-\lambda I)^{-1}$. Namely, for each $\theta \in(-\pi, \pi)$ there exist constants $R(\theta)>0$ and $C(\theta)>0$ depending on $\theta$ such that we have, for all $|\lambda| \geq R(\theta)$,

$$
\left\|(\mathfrak{A}-\lambda I)^{-1}\right\| \leq \frac{C(\theta)}{|\lambda|} .
$$

(iii) The negative axis is a direction of condensation of eigenvalues of $\mathfrak{A}$. More precisely, for each $\varepsilon>0$ there are only a finite number of eigenvalues inside the angle: $-\pi+\varepsilon<\theta<\pi-\varepsilon$.

(iv) Let

$$
N(t):=\sum_{\operatorname{Re} \lambda_{j} \geq-t} 1
$$

be the number of eigenvalues $\lambda_{j}$ such that $\operatorname{Re} \lambda_{j} \geq-t$, where each $\lambda_{j}$ is repeated according to its multiplicity. Then the following asymptotic eigenvalue distribution formula holds true:

$$
N(t)=\frac{|\Omega|}{2^{n} \pi^{n / 2} \Gamma(n / 2+1)} t^{n / 2}+o\left(t^{n / 2}\right) \quad \text { as } t \rightarrow+\infty .
$$

Here $|\Omega|$ is the Riemannian volume of the domain $\Omega$ with respect to the metric $\left(a_{i j}(x)\right)=\left(a^{i j}(x)\right)^{-1}$.

(v) The generalized eigenfunctions of $\mathfrak{A}$ are complete in the space $L^{2}(\Omega)$; they are also complete in the domain $\mathcal{D}(\mathfrak{A})$ in the $H^{2}(\Omega)$-norm.

By Theorem 2.1, the resolvent estimate (2.3) follows from the a priori estimate (2.1). Hence, it should be emphasized that assertion (ii) of Theorem 2.2 is an immediate consequence of Theorem 2.1.

The four assertions (i), (iii), (iv) and (v) solve the long-standing open problem of the asymptotic eigenvalue distribution for the homogeneous hypoelliptic Robin problem (1.3). Theorem 2.2 was proved by [26, Theorem 2] in the self-adjoint case. 
2.2 The heat asymptotics for the Robin problem (1.3)

Furthermore, we obtain from [28, Part (ii) of Theorem 1.2] with $p:=2$ that the closed operator $\mathfrak{A}$ generates a semigroup $e^{t \mathfrak{A}}$ on the space $L^{2}(\Omega)$ which is analytic in the sector

$$
\Delta_{\varepsilon}=\left\{z=t+i s: z \neq 0,|\arg z|<\frac{\pi}{2}-\varepsilon\right\}
$$

for some $0<\varepsilon<\pi / 2$. More precisely, we can define the analytic semigroup $e^{t \mathfrak{A}}$ by the contour integral formula

$$
e^{t \mathfrak{A}}=\frac{1}{2 \pi i} \int_{\gamma} e^{\lambda t}(\lambda I-\mathfrak{A})^{-1} d \lambda \quad \text { for all } t>0 .
$$

Here $\gamma$ is a path that encloses all the eigenvalues of $\mathfrak{A}$ in the resolvent set

$$
\Sigma_{\omega}=\{\lambda \in \mathbf{C}: \lambda \neq 0,|\arg \lambda| \leq \pi-\omega\}
$$

for some $0<\omega<\pi / 2$.

By assertions (i) and (iii) of Theorem 2.2, we can associate with the homogeneous Robin problem (1.3) the $\Theta$-function $\Theta(t)$ by the formula

$$
\Theta(t):=\operatorname{tr}\left(e^{t \mathfrak{A}}\right)=\sum_{j=1}^{\infty} \exp \left[\lambda_{j} t\right]
$$

By combining Iwasaki [13, Theorem II, formula (3)] with Theorem 2.2, we obtain the following trace formula as $t \downarrow 0$ :

Theorem 2.3 Assume that conditions (H.1) and (H.2) are satisfied. Then the $\Theta$ function $\Theta(t)$ has the following asymptotic expansion as $t \downarrow 0$ :

$$
\Theta(t)=\frac{1}{(4 \pi t)^{n / 2}}\left\{|\Omega|+\frac{\sqrt{\pi t}}{2}\left(|\Gamma|-2\left|\Gamma_{0}\right|\right)+o(\sqrt{t})\right\} .
$$

Here:

(1) $|\Omega|$ is the volume of $\Omega$ with respect to the metric $\left(a_{i j}(x)\right)=\left(a^{i j}(x)\right)^{-1}$.

(2) $|\Gamma|$ and $\left|\Gamma_{0}\right|$ are the surface areas of $\Gamma$ and $\Gamma_{0}$ with respect to the metric of $\Gamma$ induced by the metric $\left(a_{i j}(x)\right)$, respectively.

We give a simple example of Theorem 2.3 in a relatively compact domain $\Omega$ with smooth boundary $\Gamma$ in $\mathbf{R}^{2}$ (cf. Greiner [8, Theorem 3.2.1]):

Example 2.1 Let $\Omega$ be the unit disk with the boundary (unit circle) $\Gamma$ as in Example 1.1. If $a\left(x_{1}, x_{2}\right)$ and $b\left(x_{1}, x_{2}\right)$ are smooth functions on $\Gamma$ defined by formulas (1.4) and (1.5), respectively, then it follows that

$$
|\Omega|=\pi, \quad|\Gamma|=2 \pi, \quad\left|\Gamma_{0}\right|=\frac{\pi}{2} .
$$

We consider the Laplacian $\Delta$ with the hypoelliptic Robin boundary condition in the domain $\Omega$ with boundary $\Gamma$ :

$$
\begin{cases}\left(\frac{\partial^{2}}{\partial x_{1}^{2}}+\frac{\partial^{2}}{\partial x_{2}^{2}}+h\left(x_{1}, x_{2}\right) \frac{\partial}{\partial x_{1}}+g\left(x_{1}, x_{2}\right) \frac{\partial}{\partial x_{2}}\right) u=\mu u & \text { in } \Omega \\ a\left(x_{1}, x_{2}\right) \frac{\partial u}{\partial \mathbf{n}}+\left.\left(1-a\left(x_{1}, x_{2}\right)\right) u\right|_{\Gamma}=0 & \text { on } \Gamma .\end{cases}
$$


If the $\mu_{j}$ are the eigenvalues of the homogeneous degenerate Robin problem (2.7), then we have the asymptotic expansion formula

$$
\Theta(t)=\sum_{j=1}^{\infty} \exp \left[\mu_{j} t\right]=\frac{1}{4 t}+\frac{\sqrt{\pi}}{8 \sqrt{t}}+o\left(\frac{1}{\sqrt{t}}\right) \quad \text { as } t \downarrow 0 .
$$

\subsection{Outline of the paper}

The rest of this paper is organized as follows. In Section 3 we give sufficient conditions for the hypoellipticity of pseudo-differential operators due to Hörmander [11] (Theorem 3.1) which plays a crucial role in this paper. In Section 4 we consider non self-adjoint eigenvalue problems for a general second-order, uniformly elliptic differential operator $A$. In particular, we characterize some spectral properties of the closed realization $\mathcal{A}$ of $A$ in terms of their resolvents (Theorem 4.1), essentially due to Agmon [2, Theorems 14.4 and 15.1].

In Section 5, following Seeley [23] and [24] we formulate the homogeneous Robin problem

$$
\begin{cases}A u=f & \text { in } \Omega, \\ B \gamma u=0 & \text { on } \Gamma\end{cases}
$$

in the space $L^{2}(\Omega)$. To do so, we introduce the Poisson kernel $\mathcal{P}$ and a right inverse $\mathcal{Q}$ to the differential operator $A$ (Theorems 5.1 and 5.2). By using the operators $\mathcal{P}$ and $\mathcal{Q}$, we can define the Robin boundary operator $B \gamma$ in $L^{2}$-based Sobolev spaces (Proposition 5.1).

In Section 6, we make use of a reduction to the boundary based on the homogeneous Neumann problem (see [28, Proposition 6.10, Theorems 6.11 and 6.12] for this special reduction to the boundary). In the Neumann problem for the differential operator $A$ and its formal adjoint $A^{*}$, we have parabolic condensation of eigenvalues along the negative real axis, as discussed in Agmon [2, pp. 276-277]. Hence, we replace $A$ and $A^{*}$ by $A-\lambda_{1}$ and $A^{*}-\lambda_{1}$ for certain positive number $\lambda_{1}$ :

$$
A_{1}:=A-\lambda_{1} \text {. }
$$

Then we consider the Nemann problem

$$
\begin{cases}A_{1} v=f & \text { in } \Omega, \\ \left.\frac{\partial v}{\partial \nu}\right|_{\Gamma}=0 & \text { on } \Gamma,\end{cases}
$$

and reduce the homogeneous Robin problem

$$
\begin{cases}A_{1} u=f & \text { in } \Omega, \\ B \gamma u=a\left(x^{\prime}\right) \frac{\partial u}{\partial \boldsymbol{\nu}}+\left.b\left(x^{\prime}\right) u\right|_{\Gamma}=0 & \text { on } \Gamma\end{cases}
$$

to the study of the pseudo-differential operator $T_{1}$ on the boundary $\Gamma$ in the following way (see Proposition 6.1):

(1) The Neumann problem (2.8) has a unique solution $v=\mathcal{G}_{N} f$ in the space $H^{s+2}(\Omega)$ for every function $f \in H^{s}(\Omega)$, and the Green operator

$$
\mathcal{G}_{N}: H^{s}(\Omega) \longrightarrow H^{s+2}(\Omega)
$$

is continuous for all $s>-1 / 2$. 
(2) For every function $\varphi \in H^{s-1 / 2}(\Gamma)$, the function $w=\mathcal{P}_{1} \varphi \in H^{s}(\Omega)$ is a unique solution of the homogeneous Dirichlet problem

$$
\begin{cases}A_{1} w=0 & \text { in } \Omega, \\ \gamma_{0} w=\varphi & \text { on } \Gamma,\end{cases}
$$

and the Poisson kernel

$$
\mathcal{P}_{1}: H^{s-1 / 2}(\Gamma) \longrightarrow H^{s}(\Omega)
$$

is continuous for all $s \in \mathbf{R}$.

(3) The homogeneous Robin problem (2.9) can be reduced to the study of the pseudo-differential equation

$$
T_{1} \varphi:=B \gamma\left(\mathcal{P}_{1} \varphi\right)=-b\left(x^{\prime}\right) \gamma_{0}\left(\mathcal{G}_{N} f\right) \quad \text { on } \Gamma .
$$

The pseudo-differential equation (2.10) is a generalization of the classical Fredholm integral equation. The purpose of this section is to prove that if conditions (H.1) and (H.2) are satisfied, then the pseudo-differential operator $T_{1}$ is hypoelliptic with loss of one derivative on $\Gamma$ (Lemma 6.1).

In Section 7, by using the strong maximum principle and the Hopf boundary point lemma we prove a uniqueness theorem for the homogeneous Robin problem (2.9) (Theorem 7.1). Section 8 is devoted to the characterization of the Green operator $\mathcal{G}_{N}$ for the Neumann problem (2.8) in terms of the Boutet de Monvel calculus (Theorem 8.1). This characterization plays an important role in Section 10. In Section 9 we express explicitly the unique solution $u$ of the homogeneous Robin problem (2.9) (Theorem 9.1). Moreover, by using the Boutet de Monvel calculus we characterize the mapping property of the resolvent

$$
\mathcal{G}=\left(\mathfrak{A}-\lambda_{1} I\right)^{-1}=\mathcal{G}_{N}-\mathcal{P}_{1}\left(T_{1}^{-1} b\left(x^{\prime}\right)\left(\gamma_{0} \mathcal{G}_{N}\right)\right)
$$

for the homogeneous Robin problem (2.9) (Corollary 9.1). In Section 10 we prove Theorem 2.2, Our proof of Theorem 2.2 is based on Theorem 4.1. However, in the degenerate case we cannot use Green's formula to characterize the adjoint operator $\mathfrak{A}^{*}-\lambda_{1} I$. Therefore, we shift our attention to the adjoint $\mathcal{G}^{*}=\left(\mathfrak{A}^{*}-\lambda_{1} I\right)^{-1}$ (Theoren 10.1). More precisely, we verify all the conditions of Theorem 4.1 for the operators $\mathcal{G}$ and $\mathcal{G}^{*}$ in the framework of $L^{2}$ Sobolev spaces (see Remark 4.1).

In the last Section 11 we mention the parameter-dependent calculus of pseudodifferential boundary problems in the framework of Besov and Sobolev spaces of $L^{p}$ type due to Grubb-Kokholm [10] and Krietenstein-Schrohe [15].

\section{Hypoelliptic pseudo-differential operators}

Let $\Omega$ be an open subset of $\mathbf{R}^{n}$. A properly supported pseudo-differential operator $A$ on $\Omega$ is said to be hypoelliptic if it satisfies the condition

$$
\operatorname{sing} \operatorname{supp} u=\operatorname{sing} \operatorname{supp} A u \quad \text { for all } u \in \mathcal{D}^{\prime}(\Omega) \text {. }
$$

It is easy to see that this condition is equivalent to the following: For any open subset $\Omega_{1}$ of $\Omega$, we have the assertion

$$
u \in \mathcal{D}^{\prime}(\Omega), A u \in C^{\infty}\left(\Omega_{1}\right) \Longrightarrow u \in C^{\infty}\left(\Omega_{1}\right) .
$$


For example, elliptic operators are hypoelliptic. $4.2]$ :

The following criterion for hypoellipticity is due to Hörmander [11, Theorem

Theorem 3.1 Let $A=p(x, D) \in L_{\rho, \delta}^{m}(\Omega)$ be a properly supported, pseudo-differential operator on $\Omega$ having the complete symbol $p(x, \xi)$ with

$$
1-\rho \leq \delta<\rho \leq 1 .
$$

Assume that, for any compact $K \subset \Omega$ and any multi-indices $\alpha, \beta$ there exist constants $C_{K, \alpha, \beta}>0, C_{K}>0$ and $\mu \in \mathbf{R}$ such that we have, for all $x \in K$ and $|\xi| \geq C_{K}$,

$$
\begin{aligned}
\left|D_{\xi}^{\alpha} D_{x}^{\beta} p(x, \xi)\right| & \leq C_{K, \alpha, \beta}|p(x, \xi)|(1+|\xi|)^{-\rho|\alpha|+\delta|\beta|}, \\
|p(x, \xi)|^{-1} & \leq C_{K}(1+|\xi|)^{\mu} .
\end{aligned}
$$

Then there exists a parametrix $B \in L_{\rho, \delta}^{\mu}(\Omega)$ for $A$ :

$$
\begin{cases}A B \equiv I & \bmod L^{-\infty}(\Omega), \\ B A \equiv I & \bmod L^{-\infty}(\Omega) .\end{cases}
$$

\section{Non self-adjoint eigenvalue problems}

Let $A$ be a second-order, uniformly elliptic differential operator with real coefficients on the closure $\bar{\Omega}=\Omega \cup \Gamma$ as in Section 1 . We consider a densely defined, closed linear operator

$$
\mathcal{A}: L^{2}(\Omega) \longrightarrow L^{2}(\Omega)
$$

in $L^{2}(\Omega)$ that satisfies the following four conditions (see Agmon [2, Theorem 15.1]):

(a) The domain $\mathcal{D}(\mathcal{A})$ of definition is a subspace of $H^{2}(\Omega)$ :

$$
\mathcal{D}(\mathcal{A}) \subset H^{2}(\Omega) .
$$

(b) $\mathcal{A} u=A u$ for every $u \in \mathcal{D}(\mathcal{A})$.

Its adjoint operator

$$
\mathcal{A}^{*}: L^{2}(\Omega) \longrightarrow L^{2}(\Omega)
$$

is characterized as follows:

(c) The domain $\mathcal{D}\left(\mathcal{A}^{*}\right)$ of definition is a subspace of $H^{2}(\Omega)$ :

$$
\mathcal{D}\left(\mathcal{A}^{*}\right) \subset H^{2}(\Omega) .
$$

(d) $\mathcal{A}^{*} u=A^{\prime} u$ for every $u \in \mathcal{D}\left(\mathcal{A}^{*}\right)$.

Here $A^{\prime}$ is the formal adjoint differential operator of $A$.

Then we can prove the following spectral properties of the closed realization $\mathcal{A}$ of $A$ (see Agmon [2, Theorems 14.4 and 15.1]):

Theorem 4.1 Assume that the operator $\mathcal{A}$ satisfies the following two conditions (A) and $(B)$ : 
(A) All rays $\arg \lambda=\theta$ different from the negative axis are rays of minimal growth of the resolvent $(\mathcal{A}-\lambda I)^{-1}$. Namely, for each $\theta \in(-\pi, \pi)$ there exist constants $R(\theta)>0$ and $C(\theta)>0$ depending on $\theta$ such that we have, for all $|\lambda| \geq R(\theta)$,

$$
\left\|(\mathcal{A}-\lambda I)^{-1}\right\| \leq \frac{C(\theta)}{|\lambda|} .
$$

(B) There exists a positive integer $k$ such that

$$
\begin{aligned}
& k>\frac{n+1}{2}, \\
& \mathcal{D}\left(\mathcal{A}^{k}\right) \cup \mathcal{D}\left(\left(\mathcal{A}^{*}\right)^{k}\right) \subset H^{2 k}(\Omega) .
\end{aligned}
$$

Then we have the following four assertions:

(i) The spectrum of $\mathcal{A}$ is discrete and the eigenvalues $\lambda_{j}$ of $\mathcal{A}$ have finite multiplicities.

(ii) The negative axis is a direction of condensation of eigenvalues of $\mathcal{A}$.

(iii) If we let

$$
N(t):=\sum_{\operatorname{Re} \lambda_{j} \geq-t} 1
$$

then we have the asymptotic eigenvalue distribution formula

$$
N(t)=\frac{1}{(2 \pi)^{n}} \int_{\Omega}|A(x)| d x \cdot t^{n / 2}+o\left(t^{n / 2}\right) \quad \text { as } t \rightarrow+\infty .
$$

Here $|A(x)|$ denotes the volume of the subset

$$
A(x)=\left\{\xi \in \mathbf{R}^{n}: \sum_{i, j=1}^{n} a^{i j}(x) \xi_{i} \xi_{j}<1\right\} .
$$

(iv) The generalized eigenfunctions of $\mathcal{A}$ are complete in $L^{2}(\Omega)$; they are also complete in the domain $\mathcal{D}(\mathcal{A})$ in the $H^{2}(\Omega)$-norm.

Remark 4.1 Condition (4.2b) may be replaced by the following one (see Agmon [2, Section 15, p. 263]):

$$
\mathcal{R}\left(\mathcal{A}^{-k}\right) \cup \mathcal{R}\left(\left(\mathcal{A}^{*}\right)^{-k}\right) \subset H^{2 k}(\Omega) \text { for a positive integer } k>\frac{n+1}{2} .
$$

Indeed, it suffices to note that

$$
\mathcal{R}\left(\mathcal{A}^{-k}\right)=\mathcal{D}\left(\mathcal{A}^{k}\right), \quad \mathcal{R}\left(\left(\mathcal{A}^{*}\right)^{-k}\right)=\mathcal{D}\left(\left(\mathcal{A}^{*}\right)^{k}\right)
$$

By Theorem 2.1, we obtain that the closed operator $\mathfrak{A}$, defined by formula (2.2), satisfies condition (A) in Theorem 4.1, since the resolvent estimate (4.1) is an immediate consequence of the a priori estimate (2.1).

Therefore, we have only to show that the closed operator $\mathfrak{A}-\lambda_{1} I$ satisfies condition (4.4) for some positive number $\lambda_{1}$ in order to prove Theorem 2.2. 


\section{Formulation of the homogeneous Robin problem}

In this section we formulate the homogeneous Robin problem for second-order, uniformly elliptic differential operators in terms of pseudo-differential operators.

Following Seeley [23] and [24], we let

$$
\mathcal{N}_{0}(A):=\left\{u \in C^{\infty}(M): \operatorname{supp} u \subset \bar{\Omega}, A u=0 \text { in } \Omega\right\} .
$$

It is known (see [23, Theorem 7]) that $\mathcal{N}_{0}(A)$ is finite dimensional.

We remark that all the sufficiently large eigenvalues of the Dirichlet problem for the differential operator $A$ and its formal adjoint $A^{*}$ lie in the parabolic type region, as discussed in Agmon [2, pp. 274-277] and Mizohata [18, Chapter 3]. Hence, by considering $A-\lambda$ and $A^{*}-\lambda$ for certain positive number $\lambda$ we may assume that

$$
\mathcal{N}_{0}(A)=\mathcal{N}_{0}\left(A^{*}\right)=\{0\}
$$

Then we have the following theorem for surface potentials (see [23, Theorems 5 and 6$]$ and [24, pp. 274-275]):

Theorem 5.1 Assume that condition (5.1) is satisfied. If $s \in \mathbf{R}$, we let

$$
\mathcal{N}(A, s):=\left\{u \in H^{s}(\Omega): A u=0 \text { in } \Omega\right\} .
$$

Then the spaces $\mathcal{N}(A, s)$ and $H^{s-1 / 2}(\Gamma)$ are isomorphic in such a way that

$$
\begin{aligned}
& \mathcal{N}(A, s) \stackrel{\gamma_{0}}{\longrightarrow} H^{s-1 / 2}(\Gamma), \\
& \mathcal{N}(A, s) \overleftarrow{\mathcal{P}} H^{s-1 / 2}(\Gamma) .
\end{aligned}
$$

Here $\gamma_{0}$ is the trace operator and $\mathcal{P}$ is the Poisson kernel.

In other words, for every $\varphi \in H^{s-1 / 2}(\Gamma)$ the function $w=\mathcal{P} \varphi \in H^{s}(\Omega)$ is a unique solution of the Dirichlet problem

$$
\begin{cases}A w=0 & \text { in } \Omega, \\ \gamma_{0} w=\varphi & \text { on } \Gamma .\end{cases}
$$

Furthermore, we have the following theorem for volume potentials (see [24, pp. $276-277])$ :

Theorem 5.2 If condition (5.1) is satisfied, then there exists an elliptic, pseudodifferential operator $C$ of order -2 on $M$ such that

$$
\left.A(C E f)\right|_{\Omega}=f \quad \text { in } \Omega \text { for every } f \in H^{s}(\Omega) \text {. }
$$

Here the operator

$$
E: H^{s}(\Omega) \longrightarrow H^{s}(M)
$$

is Seeley's extension operator for each $s \in \mathbf{R}$ (see [1, Theorems 5.21 and 5.22]). 
If we introduce an operator $\mathcal{Q}$ by the formula

$$
\mathcal{Q} f=\left.C\left(f^{0}\right)\right|_{\Omega} \text { for every } f \in H^{s}(\Omega),
$$

where $f^{0}$ is the extension of $f$ by zero to the double $M$, then it follows from the transmission property of $C$ (see [21, p. 157, Theorem 7]) that

$$
\mathcal{Q}: H^{s}(\Omega) \longrightarrow H^{s+2}(\Omega)
$$

is continuous for every $s>-1 / 2$ and further from formula (5.3) that

$$
A \mathcal{Q} f=f \quad \text { in } \Omega .
$$

Namely, the operator $\mathcal{Q}$ is a right inverse to the differential operator $A$.

Now we consider a maximal domain $H_{A}(\Omega)$ for the differential operator $A$ in the space $L^{2}(\Omega)$ as follows:

$$
H_{A}(\Omega):=\left\{u \in L^{2}(\Omega): A u \in L^{2}(\Omega)\right\} .
$$

We equip the space $H_{A}(\Omega)$ with the graph norm

$$
\|u\|_{H_{A}(\Omega)}=\left(\|u\|_{L^{2}(\Omega)}^{2}+\|A u\|_{L^{2}(\Omega)}^{2}\right)^{1 / 2}
$$

Then we can express every function $u \in H_{A}(\Omega)$ uniquely in the form

$$
u=v+w
$$

where

$$
\left\{\begin{array}{l}
v=\mathcal{Q}(A u) \in H^{2}(\Omega), \\
w=u-v=\mathcal{P} \varphi \in \mathcal{N}(A, 0) .
\end{array}\right.
$$

Therefore, by using Theorem 5.1 we can define the trace mapping

$$
\gamma=\left(\gamma_{0}, \gamma_{1}\right): H_{A}(\Omega) \longrightarrow H^{-1 / 2}(\Gamma) \oplus H^{-3 / 2}(\Gamma)
$$

as follows (cf. Lions-Magenes [17]):

$$
\left\{\begin{array}{l}
\gamma_{0} u=\gamma_{0}(\mathcal{Q}(A u))+\gamma_{0} w \in H^{-1 / 2}(\Gamma), \\
\gamma_{1} u=\gamma_{1}(\mathcal{Q}(A u))+\gamma_{1} w \in H^{-3 / 2}(\Gamma) .
\end{array}\right.
$$

In this way, we can define the Robin boundary operator $B \gamma$ by the formula

$$
B \gamma u=a\left(x^{\prime}\right) \frac{\partial u}{\partial \nu}+\left.b\left(x^{\prime}\right) u\right|_{\Gamma}=a\left(x^{\prime}\right) \gamma_{1} u+b\left(x^{\prime}\right) \gamma_{0} u \quad \text { for } u \in H_{A}(\Omega) .
$$

Then we have the following:

Proposition 5.1 The Robin boundary operator

$$
B \gamma=a\left(x^{\prime}\right) \gamma_{1}+b\left(x^{\prime}\right) \gamma_{0}: H_{A}(\Omega) \longrightarrow H^{-3 / 2}(\Gamma)
$$

is continuous.

Now we can formulate the homogeneous degenerate Robin problem as follows: Given a function $f \in L^{2}(\Omega)$, find a function $u \in L^{2}(\Omega)$ such that

$$
\begin{cases}A u=f & \text { in } \Omega, \\ B \gamma u=0 & \text { on } \Gamma .\end{cases}
$$




\section{Reduction to the boundary}

In the Neumann problem for the differential operator $A$ and its formal adjoint $A^{*}$, we have parabolic condensation of eigenvalues along the negative real axis, as discussed in Agmon [2, pp. 276-277]. Hence, we can replace $A$ and $A^{*}$ by $A-\lambda_{1}$ and $A^{*}-\lambda_{1}$ for certain positive number $\lambda_{1}$ so that condition (5.1) is satisfied:

$$
\mathcal{N}_{0}\left(A-\lambda_{1}\right)=\mathcal{N}_{0}\left(A^{*}-\lambda_{1}\right)=\{0\} .
$$

Therefore, if we let

$$
A_{1}:=A-\lambda_{1},
$$

then Theorems 5.1 and 5.2 hold true for the differential operator $A_{1}$. In particular, $A_{1}$ has a right inverse $\mathcal{Q}_{1}$.

In this section, by using the Neumann problem just as in [28] we reduce the homogeneous Robin problem

$$
\begin{cases}A_{1} u=\left(A-\lambda_{1}\right) u=f & \text { in } \Omega, \\ B \gamma u=a\left(x^{\prime}\right) \frac{\partial u}{\partial \boldsymbol{\nu}}+\left.b\left(x^{\prime}\right) u\right|_{\Gamma}=0 & \text { on } \Gamma\end{cases}
$$

to the study of a first-order, pseudo-differential operator $T_{1}$ on the boundary $\Gamma$.

Step 1: First, we consider the homogeneous Neumann problem

$$
\begin{cases}A_{1} v=\left(A-\lambda_{1}\right) v=f & \text { in } \Omega, \\ \left.\frac{\partial v}{\partial \nu}\right|_{\Gamma}=0 & \text { on } \Gamma .\end{cases}
$$

It is known ([5], [7], [31]) that the Neumann problem (N) has a unique solution $v \in H^{s+2}(\Omega)$ for every function $f \in H^{s}(\Omega)$ with $s>-1 / 2$. If we let

$$
v:=\mathcal{G}_{N} f \quad \text { for } f \in H^{s}(\Omega),
$$

then it follows that the Green operator

$$
\mathcal{G}_{N}: H^{s}(\Omega) \longrightarrow H^{s+2}(\Omega)
$$

is continuous for every $s>-1 / 2$.

Step 2: If we let

$$
w:=u-v=u-\mathcal{G}_{N} f,
$$

then it follows that

$$
\begin{cases}A_{1} w=f-f=0 & \text { in } \Omega, \\ B \boldsymbol{\gamma} w=B \boldsymbol{\gamma} u-B \boldsymbol{\gamma} v=-B \boldsymbol{\gamma}\left(\mathcal{G}_{N} f\right)=-b\left(x^{\prime}\right) \gamma_{0}\left(\mathcal{G}_{N} f\right) & \text { on } \Gamma,\end{cases}
$$

since we have the formula

$$
B \gamma\left(\mathcal{G}_{N} f\right)=a\left(x^{\prime}\right) \gamma_{1}\left(\mathcal{G}_{N} f\right)+b\left(x^{\prime}\right) \gamma_{0}\left(\mathcal{G}_{N} f\right)=b\left(x^{\prime}\right) \gamma_{0}\left(\mathcal{G}_{N} f\right) \quad \text { on } \Gamma \text {. }
$$

Hence, we can express the solution $u$ of the homogeneous Robin problem (6.2) as follows:

$$
\begin{aligned}
& u=\mathcal{G}_{N} f+\mathcal{P}_{1} \varphi, \\
& B \gamma\left(\mathcal{P}_{1} \varphi\right)=-b\left(x^{\prime}\right) \gamma_{0}\left(\mathcal{G}_{N} f\right) \quad \text { on } \Gamma,
\end{aligned}
$$




$$
\varphi=\gamma_{0}\left(u-\mathcal{G}_{N} f\right) .
$$

Here $\mathcal{P}_{1}$ is the Poisson kernel for the differential operator $A_{1}$.

Step 3: If we let

$$
\begin{aligned}
T_{1}: C^{\infty}(\Gamma) & \longrightarrow C^{\infty}(\Gamma) \\
\varphi & \longmapsto B \gamma\left(\mathcal{P}_{1} \varphi\right),
\end{aligned}
$$

then we have the formula

$$
T_{1}=a\left(x^{\prime}\right) \Pi_{1}+b\left(x^{\prime}\right),
$$

where $\Pi_{1}$ is the Dirichlet-to-Neumann operator defined as follows:

$$
\Pi \varphi_{1}:=\gamma_{1}\left(\mathcal{P}_{1} \varphi\right)=\left.\frac{\partial}{\partial \nu}\left(\mathcal{P}_{1} \varphi\right)\right|_{\Gamma} \quad \text { for all } \varphi \in C^{\infty}(\Gamma)
$$

Therefore, we obtain from formulas (6.6) and (6.7) that

$$
T_{1} \varphi=\left(a\left(x^{\prime}\right) \Pi_{1}+b\left(x^{\prime}\right)\right) \varphi=-b\left(x^{\prime}\right) \gamma_{0}\left(\mathcal{G}_{N} f\right) \quad \text { on } \Gamma .
$$

Step 4: By combining formulas (6.6) and (6.8), we have proved the following fundamental proposition:

Proposition 6.1 Assume that condition (6.1) is satisfied. If $f \in H^{s}(\Omega)$ with $s>$ $-1 / 2$, then the homogeneous Robin problem (6.2) can be reduced to the study of the pseudo-differential equation (6.8). Moreover, the solutions $u$ and $\varphi$ are related as follows:

$$
\left\{\begin{array}{l}
u=\mathcal{G}_{N} f+\mathcal{P}_{1} \varphi \\
\varphi=\gamma_{0}\left(u-\mathcal{G}_{N} f\right) .
\end{array}\right.
$$

For the pseudo-differential operator $T_{1}$, we have the following fundamental lemma ([14, Theorem 3.1], [28, Lemma 7.3]):

Lemma 6.1 Assume that conditions (H.1) and (H.2) are satisfied. Then the pseudodifferential operator $T_{1}=a\left(x^{\prime}\right) \Pi_{1}+b\left(x^{\prime}\right)$ has a parametrix $S_{1}$ that belongs to the Hörmander class $L_{1,1 / 2}^{0}(\Gamma)$. Moreover, the parametrix $S_{1}$ maps $H^{s}(\Gamma)$ continuously into itself for every $s \in \mathbf{R}$.

Proof (1) First, we remark that there is a homotopy in the class of second-order uniformly elliptic symbols between $A-\lambda_{1}$ and $\Delta-1$ such that

$$
A_{t}:=t\left(A-\lambda_{1}\right)+(1-t)(\Delta-1) \quad \text { for } 0 \leq t \leq 1 .
$$

Therefore, we are reduced to the study of the differential operator

$$
A_{0}=\Delta-1=\frac{\partial^{2}}{\partial x_{1}^{2}}+\ldots+\frac{\partial^{2}}{\partial x_{n}^{2}}-1 \text {. }
$$

Then it follows from [27, Section 10.7] (cf. [6]) that the complete symbol $t\left(x^{\prime}, \xi^{\prime}\right)$ of the pseudo-differential operator $T_{1}=a\left(x^{\prime}\right) \Pi_{1}+b\left(x^{\prime}\right)$ is given by the formula

$$
t\left(x^{\prime}, \xi^{\prime}\right)=a\left(x^{\prime}\right)\left|\xi^{\prime}\right|+\left[b\left(x^{\prime}\right)+\frac{a\left(x^{\prime}\right)}{2}\left(\frac{\omega_{x^{\prime}}\left(\widehat{\xi^{\prime}}, \widehat{\xi}^{\prime}\right)}{\left|\xi^{\prime}\right|^{2}}-(n-1) M\left(x^{\prime}\right)\right)\right]
$$




$$
\begin{aligned}
& -\sqrt{-1} \frac{a\left(x^{\prime}\right)}{2}\left(\operatorname{div} \delta_{\left(\xi^{\prime}\right)}\left(x^{\prime}\right)\right)+\text { terms of order } \leq-1 \\
:= & t_{0}\left(x^{\prime}, \xi^{\prime}\right)+\text { terms of order } \leq-1,
\end{aligned}
$$

where

$$
\begin{aligned}
t_{0}\left(x^{\prime}, \xi^{\prime}\right)= & a\left(x^{\prime}\right)\left|\xi^{\prime}\right|+\left[b\left(x^{\prime}\right)+\frac{a\left(x^{\prime}\right)}{2}\left(\frac{\omega_{x^{\prime}}\left(\widehat{\xi}^{\prime}, \widehat{\xi}^{\prime}\right)}{\left|\xi^{\prime}\right|^{2}}-(n-1) M\left(x^{\prime}\right)\right)\right] \\
& -\sqrt{-1} \frac{a\left(x^{\prime}\right)}{2}\left(\operatorname{div} \delta_{\left(\xi^{\prime}\right)}\left(x^{\prime}\right)\right) .
\end{aligned}
$$

(2) Secondly, by letting

$$
\begin{aligned}
& \widetilde{a}\left(x^{\prime}\right)=\frac{a\left(x^{\prime}\right)}{a\left(x^{\prime}\right)+b\left(x^{\prime}\right)}, \\
& \widetilde{b}\left(x^{\prime}\right)=\frac{b\left(x^{\prime}\right)}{a\left(x^{\prime}\right)+b\left(x^{\prime}\right)},
\end{aligned}
$$

we may assume that the Robin boundary condition $B \gamma$ is of the form

$$
B \boldsymbol{\gamma} u=a\left(x^{\prime}\right) \frac{\partial u}{\partial \boldsymbol{\nu}}+\left.\left(1-a\left(x^{\prime}\right)\right) u\right|_{\Gamma}=0 \quad \text { on } \Gamma,
$$

where

$$
0 \leq a\left(x^{\prime}\right) \leq 1 \quad \text { on } \Gamma .
$$

Thus we remark that formula (6.9) can be simplified as follows:

$$
\begin{aligned}
t_{0}\left(x^{\prime}, \xi^{\prime}\right)= & a\left(x^{\prime}\right)\left|\xi^{\prime}\right|+\left[\left(1-a\left(x^{\prime}\right)\right)+\frac{a\left(x^{\prime}\right)}{2}\left(\frac{\omega_{x^{\prime}}\left(\widehat{\xi}^{\prime}, \widehat{\xi}^{\prime}\right)}{\left|\xi^{\prime}\right|^{2}}-(n-1) M\left(x^{\prime}\right)\right)\right] \\
& -\sqrt{-1} \frac{a\left(x^{\prime}\right)}{2}\left(\operatorname{div} \delta_{\left(\xi^{\prime}\right)}\left(x^{\prime}\right)\right) .
\end{aligned}
$$

Then it is easy to see that the principal part

$$
s_{0}\left(x^{\prime}, \xi^{\prime}\right)=\frac{1}{t_{0}\left(x^{\prime}, \xi^{\prime}\right)}
$$

of the inverse $1 / t\left(x^{\prime}, \xi^{\prime}\right)$ satisfies the inequality

$$
\begin{aligned}
& \left|s_{0}\left(x^{\prime}, \xi^{\prime}\right)\right|=\frac{1}{\left|t_{0}\left(x^{\prime}, \xi^{\prime}\right)\right|} \\
& \leq \begin{cases}\frac{1}{1-\delta_{1}} & \text { for }\left|\xi^{\prime}\right| \text { sufficiently large if } 0 \leq a\left(x^{\prime}\right) \leq \delta_{1} \text { for some } 0<\delta_{1}<1, \\
\frac{1}{\delta_{1}} & \text { for }\left|\xi^{\prime}\right| \text { sufficiently large if } a\left(x^{\prime}\right) \geq \delta_{1} .\end{cases}
\end{aligned}
$$

Therefore, we find from inequality (6.10) (see [28, Lemma 7.2]) that the principal part $s_{0}\left(x^{\prime}, \xi^{\prime}\right)$ satisfies conditions (3.1a) and (3.1b) with

$$
\mu:=0, \quad \rho:=1, \quad \delta:=\frac{1}{2} .
$$

This proves that the pseudo-differential operator $T_{1}$ has a parametrix

$$
S_{1} \in L_{1,1 / 2}^{0}(\Gamma) .
$$

Finally, the boundedness of the parametrix $S_{1}$ follows from an application of Bourdaud [3, Theorem 1] with $\delta:=1 / 2$ (cf. [27, Appendix A]).

The proof of Lemma 6.1 is complete. 


\section{Uniqueness theorem for the Robin problem}

In this section we consider the following homogeneous Robin problem under condition (6.1): Given a function $f(x)$ defined in $\Omega$, find a function $u(x)$ in $\Omega$ such that

$$
\begin{cases}A_{1} u=\left(A-\lambda_{1}\right) u=f & \text { in } \Omega, \\ B \gamma u=a\left(x^{\prime}\right) \frac{\partial u}{\partial \boldsymbol{\nu}}+\left.b\left(x^{\prime}\right) u\right|_{\Gamma}=0 & \text { on } \Gamma .\end{cases}
$$

The purpose of this section is to prove the following uniqueness theorem for the Robin problem (6.2):

Theorem 7.1 If conditions (H.1) and (H.2) are satisfied, then every solution $u \in$ $C^{2}(\bar{\Omega})$ of the Robin problem

$$
\begin{cases}A_{1} u=\left(A-\lambda_{1}\right) u=0 & \text { in } \Omega, \\ B \gamma u=a\left(x^{\prime}\right) \frac{\partial u}{\partial \boldsymbol{\nu}}+\left.b\left(x^{\prime}\right) u\right|_{\Gamma}=0 & \text { on } \Gamma\end{cases}
$$

is identically equal to zero in $\Omega$ :

$$
u(x) \equiv 0 \quad \text { in } \Omega .
$$

Proof (1) If a solution $u(x)$ of the Robin problem (7.1) is a constant $m$ in $\Omega$, then it follows that

$$
0=\left(A-\lambda_{1}\right) u=\left(c(x)-\lambda_{1}\right) m \text { in } \Omega .
$$

This proves the desired assertion (7.2), since $c(x)-\lambda_{1} \leq-\lambda_{1}<0$ in $\Omega$.

(2) The case where $u(x)$ is not constant in $\Omega$. Our proof is based on a reduction to absurdity. We assume, to the contrary, that a solution $u \in C^{2}(\bar{\Omega})$ of the Robin problem (7.1) is not identically equal to zero in $\Omega$ :

$$
u(x) \not \equiv 0 \text { in } \Omega \text {. }
$$

Then, by considering $-u$ if necessarily we may assume that

$$
m=\max _{x \in \bar{\Omega}} u(x)>0 .
$$

By applying the strong maximum principle ([20, Chapter 2, Section 3, Theorem 5]) to the differential operator $A-\lambda_{1}$, we obtain that there exists a point $x_{0}^{\prime}$ of $\Gamma$ such that

$$
\left\{\begin{array}{l}
u\left(x_{0}^{\prime}\right)=m>0, \\
u(x)<u\left(x_{0}^{\prime}\right) \text { for all } x \in \Omega .
\end{array}\right.
$$

Furthermore, it follows from an application of the Hopf boundary point lemma ([20, Chapter 2, Section 3, Theorem 7]) that

$$
\frac{\partial u}{\partial \boldsymbol{\nu}}\left(x_{0}^{\prime}\right)>0 .
$$

However, we have the formula

$$
B \gamma u\left(x_{0}^{\prime}\right)=a\left(x_{0}^{\prime}\right) \frac{\partial u}{\partial \boldsymbol{\nu}}\left(x_{0}^{\prime}\right)+b\left(x_{0}^{\prime}\right) u\left(x_{0}^{\prime}\right)=0 .
$$

Hence, we have, by condition (H.1) and inequalities (7.3) and (7.4),

$$
a\left(x_{0}^{\prime}\right)=b\left(x_{0}^{\prime}\right)=0 .
$$

This contradicts condition (H.2).

The proof of Theorem 7.1 is now complete. 
8 The Neumann problem via the Boutet de Monvel calculus

In this section, we study the Green operator for the Neumann problem from the viewpoint of the Boutet de Monvel calculus.

For every function $f \in H^{s}(\Omega)$ with $s>-1 / 2$, the function $v=\mathcal{G}_{N} f \in H^{s+2}(\Omega)$ is the unique solution of the Neumann problem (6.3) for the elliptic differential operator $A_{1}=A-\lambda_{1}$. By applying Proposition 6.1 and Theorem 7.1 to the Neumann case $\left(a\left(x^{\prime}\right) \equiv 1\right.$ and $b\left(x^{\prime}\right) \equiv 0$ on $\left.\Gamma\right)$, we find that the Green operator $\mathcal{G}_{N}$, defined by formula (6.4), can be written in the form

$$
\mathcal{G}_{N} f=\mathcal{Q}_{1} f-\mathcal{P}_{1}\left(\Pi_{1}^{-1}\left(\gamma_{1}\left(\mathcal{Q}_{1} f\right)\right)\right) \quad \text { for } f \in H^{s}(\Omega) .
$$

Here:

(i) $\mathcal{Q}_{1}$ is a right inverse to the operator $A_{1}=A-\lambda_{1}$.

(ii) $\mathcal{P}_{1}$ is the Poisson kernel for the operator $A_{1}$.

(iii) $\Pi_{1}^{-1}$ is the inverse of the Dirichlet-to-Neumann operator $\Pi_{1}=\gamma_{1} \mathcal{P}_{1}$.

The purpose of this section is to prove the mapping property of the Green operator $\mathcal{G}_{N}$, as is shown in Tabel 8.1.

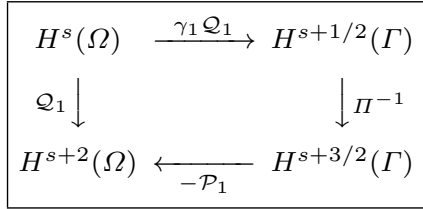

Table 8.1 The mapping property of the Green operator $\mathcal{G}_{N}$

First, the next theorem characterizes the Green operator $\mathcal{G}_{N}$ in terms of the Boutet de Monvel calculus:

Theorem 8.1 Assume that condition (6.1) is satisfied. Then the components of the Green operator $\mathcal{G}_{N}$ can be expressed in the matrix form

$$
\mathcal{N}=\left(\begin{array}{cc}
\mathcal{Q}_{1} & -\mathcal{P}_{1} \\
\gamma_{1} \mathcal{Q}_{1} & \Pi_{1}^{-1}
\end{array}\right),
$$

and the principal symbol $\sigma(\mathcal{N})$ is homotopic to the following:

$$
\left(\begin{array}{cc}
-\frac{1}{\left\langle\xi^{\prime}\right\rangle^{2}+\nu^{2}} & -\frac{1}{\left\langle\xi^{\prime}\right\rangle+i \nu} \\
\frac{1}{2} \frac{1}{\left\langle\xi^{\prime}\right\rangle-i \tau} & \frac{1}{\left\langle\xi^{\prime}\right\rangle}
\end{array}\right) .
$$

Here and in the following

$$
\begin{aligned}
& \xi=\left(\xi^{\prime}, \nu\right)=\left(\xi_{1}, \xi_{2}, \ldots, \xi_{n-1}, \nu\right) \in \mathbf{R}^{n}, \\
& \left\langle\xi^{\prime}\right\rangle=\sqrt{\left|\xi^{\prime}\right|^{2}+1} \\
& \xi=\left(\xi^{\prime}, \nu\right)=\left(\xi_{1}, \xi_{2}, \ldots, \xi_{n-1}, \nu\right) \in \mathbf{R}^{n} \quad \text { for potential operators, } \\
& \xi=\left(\xi^{\prime}, \tau\right)=\left(\xi_{1}, \xi_{2}, \ldots, \xi_{n-1}, \tau\right) \in \mathbf{R}^{n} \quad \text { for trace operators. }
\end{aligned}
$$


Proof By a homotopy argument, we are reduced to the study of the following Neumann problem

$$
\begin{cases}(\Delta-1) u=f & \text { in } \Omega, \\ \left.\frac{\partial v}{\partial \mathbf{n}}\right|_{\Gamma}=0 & \text { on } \Gamma .\end{cases}
$$

Indeed, there is a homotopy in the class of second-order uniformly elliptic symbols between $A-\lambda_{1}$ and $\Delta-1$ such that

$$
A_{t}:=t\left(A-\lambda_{1}\right)+(1-t)(\Delta-1) \quad \text { for } 0 \leq t \leq 1 .
$$

(1) We remark that the differential operator

$$
A_{0}=\Delta-1
$$

has a right inverse $\mathcal{Q}_{0}$ defined by the formula (see formula (5.4))

$$
\mathcal{Q}_{0} f(x)=\left.\left(\left(-G_{2}\right) * f^{0}\right)\right|_{\Omega}=-\int_{\Omega} G_{2}(x-y) f(y) d y \quad \text { for } f \in H^{s}(\Omega) .
$$

Here (cf. [25, Chapter V, Section 3])

$$
\begin{aligned}
& G_{2}(x)=\frac{1}{(4 \pi)^{n / 2}} \int_{0}^{\infty} e^{-t-\frac{|x|^{2}}{4 t}} t^{\frac{2-n}{2}} \frac{d t}{t} \\
& \widehat{G}_{2}(\xi)=\int_{\mathbf{R}^{n}} e^{-i x \xi} G_{2}(x) d x=\frac{1}{1+|\xi|^{2}} .
\end{aligned}
$$

Hence it follows from formula (8.5) that the principal symbol of $\mathcal{Q}_{0}$ can be written in the form

$$
-\frac{1}{1+|\xi|^{2}}=-\frac{1}{\left\langle\xi^{\prime}\right\rangle^{2}+\nu^{2}}=-\frac{1}{2\left\langle\xi^{\prime}\right\rangle}\left(\frac{1}{\left\langle\xi^{\prime}\right\rangle+i \nu}+\frac{1}{\left\langle\xi^{\prime}\right\rangle-i \nu}\right) .
$$

This is homotopic to the principal symbol of the right inverse $\mathcal{Q}_{1}$ to $A_{1}$. Hence, we obtain from the transmission property of $\mathcal{Q}_{1}$ (see [21, p. 159, Theorem 7]) that the operator

$$
\mathcal{Q}_{1}: H^{s}(\Omega) \longrightarrow H^{s+2}(\Omega)
$$

is continuous for every $s>-1 / 2$.

(2) By using Rempel-Schulze [21, p. 100, Lemma 4], we obtain from formula (8.6) that the operator $\gamma_{1} \mathcal{Q}_{0}$ is a trace operator of order -1 and type 0 with principal symbol

$$
\frac{1}{2} \frac{1}{\left\langle\xi^{\prime}\right\rangle-i \tau}
$$

This is homotopic to the principal symbol of $\gamma_{1} \mathcal{Q}_{1}$. Hence, it follows from an application of $[21$, p. 158, Theorem 5] that the trace operator

$$
\gamma_{1} \mathcal{Q}_{1}: H^{s}(\Omega) \longrightarrow H^{s+1 / 2}(\Gamma)
$$

is continuous for every $s>-1 / 2$.

(3) On the other hand, by using Rempel-Schulze [21, p. 102, Proposition 6] we find from formula (8.6) that the Poisson kernel $\mathcal{P}_{0}$ for $A_{0}$ is a potential operator of order -1 with principal symbol

$$
\frac{1}{\left\langle\xi^{\prime}\right\rangle+i \nu}
$$


This is homotopic to the principal symbol of $\mathcal{P}_{1}$ for $A_{1}$. Hence, it follows from an application of $[21$, p. 156 , Theorem 4$]$ that the potential operator

$$
\mathcal{P}_{1}: H^{s+3 / 2}(\Gamma) \longrightarrow H^{s+2}(\Omega)
$$

is continuous for every $s \in \mathbf{R}$.

(4) Finally, it follows from formula (6.9) (with $a\left(x^{\prime}\right) \equiv 1$ and $b\left(x^{\prime}\right) \equiv 0$ on $\Gamma$ ) that the principal symbol of the Dirichlet-to-Neumann operator $\Pi_{0}=\gamma_{1} \mathcal{P}_{0}$ for $A_{0}$ is given by the formula

$$
\left\langle\xi^{\prime}\right\rangle=\sqrt{\left|\xi^{\prime}\right|^{2}+1}
$$

This is homotopic to the principal symbol of the Dirichlet-to-Neumann operator $\Pi_{1}=\gamma_{1} \mathcal{P}_{1}$ for $A_{1}$. Hence its inverse

$$
\Pi_{1}^{-1}: H^{s+1 / 2}(\Gamma) \longrightarrow H^{s+3 / 2}(\Gamma)
$$

is continuous for every $s \in \mathbf{R}$.

Summing up, we find from formulas (8.6) through (8.9) that the principal symbol of the Green operator

$$
\mathcal{G}_{N}=\mathcal{Q}_{1}-\mathcal{P}_{1}\left(\Pi_{1}^{-1}\left(\gamma_{1} \mathcal{Q}_{1}\right)\right)
$$

is homotopic to the following:

$$
\begin{aligned}
& -\frac{1}{\left\langle\xi^{\prime}\right\rangle^{2}+\nu^{2}}-\left(\frac{1}{\left\langle\xi^{\prime}\right\rangle+i \nu}\right) \frac{1}{\left\langle\xi^{\prime}\right\rangle}\left(\frac{1}{2} \frac{1}{\left\langle\xi^{\prime}\right\rangle-i \tau}\right) \\
= & -\frac{1}{\left\langle\xi^{\prime}\right\rangle^{2}+\nu^{2}}-\frac{1}{2\left\langle\xi^{\prime}\right\rangle} \frac{1}{\left\langle\xi^{\prime}\right\rangle+i \nu} \frac{1}{\left\langle\xi^{\prime}\right\rangle-i \tau} .
\end{aligned}
$$

More precisely, the second term

$$
\mathcal{P}_{1}\left(\Pi_{1}^{-1}\left(\gamma_{1} \mathcal{Q}_{1}\right)\right)
$$

is called a singular Green operator of order -3 and type 0 (see [21, p. 129, Proposition 2]), and it follows from an application of Rempel-Schulze [21, p. 159, Theorem 6 ] that the operator

$$
\mathcal{P}_{1}\left(\Pi_{1}^{-1}\left(\gamma_{1} \mathcal{Q}_{1}\right)\right): H^{s}(\Omega) \longrightarrow H^{s+2}(\Omega)
$$

is continuous for every $s>-1 / 2$.

Summing up, we have proved that $\mathcal{G}_{N}$ maps $H^{s}(\Omega)$ continuously into $H^{s+2}(\Omega)$ for every $s \geq 0$, as is shown in Table 8.1.

The proof of Theorem 8.1 is complete.

Remark 8.1 By using Rempel-Schulze [21, p. 100, Lemma 4], we obtain from formula (8.6) that the operator $\gamma_{0} \mathcal{Q}_{0}$ for $A_{0}$ is a trace operator of order -2 and type 0 with principal symbol

$$
-\frac{1}{2\left\langle\xi^{\prime}\right\rangle} \frac{1}{\left\langle\xi^{\prime}\right\rangle-i \tau}
$$

This is homotopic to the principal symbol of $\gamma_{0} \mathcal{Q}_{1}$ for $A_{1}$. Hence, it follows from an application of $[21$, p. 158 , Theorem 5$]$ that the trace operator

$$
\gamma_{0} \mathcal{Q}_{1}: H^{s}(\Omega) \longrightarrow H^{s+3 / 2}(\Gamma)
$$

is continuous for every $s>-3 / 2$. 
9 Representation formula of the resolvent via the Boutet de Monvel calculus

In this section we characterize the resolvent of the homogeneous Robin problem (6.2) in terms of the Boutet de Monvel calculus (Theorem 9.1 and Corollary 9.1). This section is the heart of the subject.

Step (1): First, by combining Theorem 2.1 and Rellich's theorem we obtain that $\mathfrak{A}_{1}$ is a Fredholm operator with

$$
\operatorname{ind} \mathfrak{A}_{1}=\operatorname{ind}\left(\mathfrak{A}-\lambda_{1} I\right)=0 .
$$

Step (2): Secondly, by using Proposition 6.1 we can associate with the pseudodifferential equation (6.8) a densely defined, closed linear operator

$$
\mathcal{T}_{1}: H^{s+3 / 2}(\Gamma) \longrightarrow H^{s+3 / 2}(\Gamma)
$$

as follows.

$(\alpha)$ The domain $\mathcal{D}\left(\mathcal{T}_{1}\right)$ of definition is the space

$$
\mathcal{D}\left(\mathcal{T}_{1}\right)=\left\{\varphi \in H^{s+3 / 2}(\Gamma): T_{1} \varphi \in H^{s+3 / 2}(\Gamma)\right\} .
$$

( $\beta) \mathcal{T}_{1} \varphi=T_{1} \varphi=\left(a\left(x^{\prime}\right) \Pi_{1}+b\left(x^{\prime}\right)\right) \varphi$ for every $\varphi \in \mathcal{D}\left(\mathcal{T}_{1}\right)$.

Indeed, since $T_{1}: H^{s+3 / 2}(\Gamma) \rightarrow H^{s+1 / 2}(\Gamma)$ is continuous, it follows that $\mathcal{T}_{1}$ is a closed operator. Furthermore, the operator $\mathcal{T}_{1}$ is densely defined, since the domain $\mathcal{D}\left(\mathcal{T}_{1}\right)$ contains a dense subspace $C^{\infty}(\Gamma)$ of $H^{s+3 / 2}(\Gamma)$.

The adjoint operator $\mathcal{T}_{1}{ }^{*}$ of $\mathcal{T}_{1}$ is a densely defined, closed linear operator

$$
\mathcal{T}_{1}{ }^{*}: H^{-s-3 / 2}(\Gamma) \longrightarrow H^{-s-3 / 2}(\Gamma)
$$

such that

$$
\begin{aligned}
s+3 / 2\left(\mathcal{T}_{1} \varphi, \psi\right)_{-s-3 / 2}= & { }_{s+3 / 2}\left(\varphi, \mathcal{T}_{1}{ }^{*} \psi\right)_{-s-3 / 2} \\
& \quad \text { for all } \varphi \in \mathcal{D}\left(\mathcal{T}_{1}\right) \text { and } \psi \in \mathcal{D}\left(\mathcal{T}_{1}{ }^{*}\right) .
\end{aligned}
$$

Here $\sigma(\cdot, \cdot)_{-\sigma}$ is the sesquilinear pairing between the Sobolev spaces $H^{\sigma}(\Gamma)$ and $H^{-\sigma}(\Gamma)$ for $\sigma \in \mathbf{R}$.

However, by using Lemma 6.1 we can prove the following regularity results:

(i) If $\varphi \in \mathcal{D}^{\prime}(\Gamma)$ and $T_{1} \varphi \in H^{\sigma}(\Gamma)$ for $\sigma \in \mathbf{R}$, then it follows that $\varphi \in H^{\sigma}(\Gamma)$. In particular, we have the regularity result for the null space of $\mathcal{T}_{1}$ :

$$
\mathcal{N}\left(\mathcal{T}_{1}\right)=\left\{\varphi \in H^{s+3 / 2}(\Gamma): T_{1} \varphi=0\right\} \subset C^{\infty}(\Gamma) .
$$

(ii) If $\psi \in \mathcal{D}^{\prime}(\Gamma)$ and $T_{1}^{*} \psi \in H^{\sigma}(\Gamma)$ for $\sigma \in \mathbf{R}$, then it follows that $\psi \in H^{\sigma}(\Gamma)$. In particular, we have the regularity result for the null space of $\mathcal{T}_{1}{ }^{*}$ :

$$
\mathcal{N}\left(\mathcal{T}_{1}{ }^{*}\right)=\left\{\psi \in H^{-s-3 / 2}(\Gamma): T_{1}^{*} \psi=0\right\} \subset C^{\infty}(\Gamma) .
$$

Here $T_{1}{ }^{*}$ is the adjoint of the pseudo-differential operator $T_{1}=a\left(x^{\prime}\right) \Pi_{1}+b\left(x^{\prime}\right)$. 
Therefore, we obtain from assertions (9.2) and (9.3) that the operator $\mathcal{T}_{1}$ is a Fredholm operator whose index is indendent of $s \in \mathbf{R}$ :

$$
\operatorname{ind} \mathcal{T}_{1}=\operatorname{dim} \mathcal{N}\left(\mathcal{T}_{1}\right)-\operatorname{dim} \mathcal{N}\left(\mathcal{T}_{1}{ }^{*}\right)
$$

In this way, by using Proposition 6.1 with $s:=0$ we obtain from assertion (9.1) that

$$
\text { ind } \mathcal{T}_{1}=\text { ind } \mathfrak{A}_{1}=0 \text {. }
$$
that

Step (3) On the other hand, it follows from Proposition 6.1 and Theorem 7.1

$$
\mathcal{N}\left(\mathcal{T}_{1}\right)=\{0\}
$$

Summing up, we obtain from assertions (9.4) and (9.5) that the operator

$$
\mathcal{T}_{1}: H^{s+3 / 2}(\Gamma) \longrightarrow H^{s+3 / 2}(\Gamma)
$$

is bijective for every $s \in \mathbf{R}$.

Step (4): Now we are in a position to prove the following fundamental theorem:

Theorem 9.1 Assume that conditions (H.1) and (H.2) are satisfied. If $f \in H^{s}(\Omega)$ with $s>-1 / 2$, then every solution $u \in L^{2}(\Omega)$ of the homogeneous Robin problem (6.2) belongs to the space $H^{s+2}(\Omega)$. Moreover, every solution $u \in H^{s+2}(\Omega)$ can be uniquely expressed in the form

$$
u=\mathcal{G} f:=\mathcal{G}_{N} f-\mathcal{P}_{1}\left(\mathcal{T}_{1}{ }^{-1} b\left(x^{\prime}\right)\left(\gamma_{0}\left(\mathcal{G}_{N} f\right)\right)\right)
$$

Here:

(i) $\mathcal{G}_{N}: H^{s}(\Omega) \rightarrow H^{s+2}(\Omega)$ is the Green operator for the Neumann problem (6.3).

(ii) $\mathcal{P}_{1}: H^{s+3 / 2}(\Gamma) \rightarrow H^{s+2}(\Omega)$ is the Poisson kernel for the differential operator $A_{1}$.

(iii) $\mathcal{T}_{1}^{-1}$ is the inverse of the operator $\mathcal{T}_{1}$ in the Sobolev space $H^{s+3 / 2}(\Gamma)$.

Proof By combining Theorem 7.1 and Proposition 6.1 we find that every solution $u \in H^{s+2}(\Omega)$ can be uniquely expressed by formula (9.7).

(1) First, by formula (8.1) it follows that

$$
\gamma_{0} \mathcal{G}_{N}=\gamma_{0}\left(\mathcal{Q}_{1}-\mathcal{P}_{1}\left(\Pi_{1}^{-1}\left(\gamma_{1} \mathcal{Q}_{1}\right)\right)\right)=\gamma_{0} \mathcal{Q}_{1}-\Pi_{1}^{-1}\left(\gamma_{1} \mathcal{Q}_{1}\right)
$$

Hence we find from formulas (8.11), (8.9) and (8.7) that the principal symbol of $\gamma_{0} \mathcal{G}_{N}$ is homotopic to the following:

$$
-\frac{1}{2\left\langle\xi^{\prime}\right\rangle} \frac{1}{\left\langle\xi^{\prime}\right\rangle-i \tau}-\frac{1}{\left\langle\xi^{\prime}\right\rangle} \frac{1}{2} \frac{1}{\left\langle\xi^{\prime}\right\rangle-i \tau}=-\frac{1}{\left\langle\xi^{\prime}\right\rangle} \frac{1}{\left\langle\xi^{\prime}\right\rangle-i \tau} .
$$

This proves that the operator $\gamma_{0} \mathcal{G}_{N}$ is a trace operator of order -2 and type 0 . Hence, it follows from an application of Rempel-Schulze [21, p. 158, Theorem 5] that the trace operator

$$
\gamma_{0} \mathcal{G}_{N}: H^{s}(\Omega) \longrightarrow H^{s+3 / 2}(\Gamma)
$$

is continuous for every $s>-3 / 2$. 
(2) Secondly, it follows from Lemma 6.1 that the operator $T_{1}^{-1} b\left(x^{\prime}\right)$ is a pseudodifferential operator in $L_{1,1 / 2}^{0}(\Gamma)$ with the principal part which is homotopic to the following (see formula $(6.9)$ ):

$$
b\left(x^{\prime}\right) s_{0}\left(x^{\prime}, \xi^{\prime}\right)=\frac{b\left(x^{\prime}\right)}{t_{0}\left(x^{\prime}, \xi^{\prime}\right)} .
$$

Hence, by applying Bourdaud [3, Theorem 1] with $\delta:=1 / 2$ we obtain from assertion (9.6) that the operator

$$
\mathcal{T}_{1}{ }^{-1} b\left(x^{\prime}\right): H^{s+3 / 2}(\Gamma) \longrightarrow H^{s+3 / 2}(\Gamma)
$$

is continuous for every $s \in \mathbf{R}$.

The proof of Theorem 9.1 is complete.

$$
\begin{array}{|cc|}
\hline H^{s}(\Omega) & \stackrel{\gamma_{0} \mathcal{G}_{N}}{\longrightarrow} H^{s+3 / 2}(\Gamma) \\
\mathcal{G}_{N} \downarrow & \mathcal{T}_{1}^{-1} b\left(x^{\prime}\right) \downarrow \\
H^{s+2}(\Omega) & \longleftarrow \\
\hline
\end{array}
$$

Table 9.1 The mapping property of the resolvent $\mathcal{G}=\left(\mathfrak{A}-\lambda_{1} I\right)^{-1}$

By combining Theorems 8.1 and 9.1, we have the following:

Corollary 9.1 If conditions (H.1) and (H.2) are satisfied, then the resolvent

$$
\mathcal{G}=\left(\mathfrak{A}-\lambda_{1} I\right)^{-1}=\mathcal{G}_{N}-\mathcal{P}_{1}\left(\mathcal{T}_{1}{ }^{-1} b\left(x^{\prime}\right)\left(\gamma_{0} \mathcal{G}_{N}\right)\right)
$$

maps $H^{s}(\Omega)$ continuously into $H^{s+2}(\Omega)$ for every $s \geq 0$, as is shown in Table 9.1. Moreover, the components of the operator $\mathcal{G}$ can be written in the matrix form

$$
\mathcal{R}=\left(\begin{array}{cc}
\mathcal{G}_{N} & -\mathcal{P}_{1} \\
\gamma_{0} \mathcal{G}_{N} & \mathcal{T}_{1}^{-1} b\left(x^{\prime}\right)
\end{array}\right)
$$

and the principal symbol $\sigma(\mathcal{R})$ of $\mathcal{R}$ is homotopic to the following:

$$
\left(\begin{array}{cc}
-\frac{1}{\left\langle\xi^{\prime}\right\rangle^{2}+\nu^{2}}-\frac{1}{2\left\langle\xi^{\prime}\right\rangle} \frac{1}{\left\langle\xi^{\prime}\right\rangle+i \nu} \frac{1}{\left\langle\xi^{\prime}\right\rangle-i \tau} & -\frac{1}{\left\langle\xi^{\prime}\right\rangle+i \nu} \\
-\frac{1}{\left\langle\xi^{\prime}\right\rangle} \frac{1}{\left\langle\xi^{\prime}\right\rangle-i \tau} & \frac{b\left(x^{\prime}\right)}{t_{0}\left(x^{\prime}, \xi^{\prime}\right)}
\end{array}\right) .
$$

\section{Proof of Theorem 2.2 via the Boutet de Monvel calculus}

In this section we prove Theorem 2.2, by using the Boutet de Monvel calculus (see [4], [21], [22]). Our proof of Theorem 2.2 is based on Theorem 4.1 and Remark 4.1 with

$$
\mathcal{A}:=\mathfrak{A}-\lambda_{1} I
$$

under condition (6.1). More precisely, we have only to verify condition (4.4) for the resolvent $\left(\mathfrak{A}-\lambda_{1} I\right)^{-1}$ given by the representation formula (9.7). The proof of Theorem 2.2 is divided into two subsections. 
10.1 The mapping property of the adjoint operator $\mathcal{G}^{*}$

We are in a position to characterize the mapping property of the adjoint $\mathcal{G}^{*}$ of the resolvent $\mathcal{G}=\left(\mathfrak{A}-\lambda_{1} I\right)^{-1}$ in the framework of Sobolev spaces of $L^{2}$ type:

Theorem 10.1 Assume that conditions (H.1) and (H.2) are satisfied. If $\mathcal{G}=(\mathfrak{A}-$ $\left.\lambda_{1} I\right)^{-1}$ is the resolvent given by formula (9.7), then its adjoint operator

$$
\mathcal{G}^{*}=\left(\mathfrak{A}^{*}-\lambda_{1} I\right)^{-1}=\left(\mathcal{G}_{N}\right)^{*}-\left(\gamma_{0} \mathcal{G}_{N}\right)^{*}\left(\mathcal{T}_{1}{ }^{-1} b\left(x^{\prime}\right)\right)^{*}\left(\mathcal{P}_{1}\right)^{*}
$$

maps $H^{s}(\Omega)$ continuously into $H^{s+2}(\Omega)$ for every $s \geq 0$, as is shown in Table 10.1.

$$
\begin{gathered}
H^{s}(\Omega) \stackrel{-\left(\mathcal{P}_{1}\right)^{*}}{\longrightarrow} H^{s+1 / 2}(\Gamma) \\
\downarrow\left(\mathcal{G}_{N}\right)^{*} \quad\left(\mathcal{T}_{1}^{-1} b\left(x^{\prime}\right)\right)^{*} \downarrow \\
H^{s+2}(\Omega) \overleftarrow{\left(\gamma_{0} \mathcal{G}_{N}\right)^{*}} H^{s+1 / 2}(\Gamma)
\end{gathered}
$$

Table 10.1 The mapping property of the adjoint operator $\mathcal{G}^{*}=\left(\mathfrak{A}^{*}-\lambda_{1} I\right)^{-1}$

Proof (1) First, it follows from formulas (9.11) and (9.12) that the principal symbol of Green operator $\mathcal{G}_{N}$ is homotopic to the following:

$$
-\frac{1}{\left\langle\xi^{\prime}\right\rangle^{2}+\nu^{2}}-\frac{1}{2\left\langle\xi^{\prime}\right\rangle} \frac{1}{\left\langle\xi^{\prime}\right\rangle+i \nu} \frac{1}{\left\langle\xi^{\prime}\right\rangle-i \tau} .
$$

By using Rempel-Schulze [21, p. 102, Proposition 6 and p. 151, Proposition 10], we find from formula $(10.2)$ that the principal symbol of the adjoint $\left(\mathcal{G}_{N}\right)^{*}$ is given by the same formula (10.2). Therefore, it follows from an application of [21, p. 159, Theorems 6 and 7] that the adjoint

$$
\left(\mathcal{G}_{N}\right)^{*}: H^{s}(\Omega) \longrightarrow H^{s+2}(\Omega)
$$

is continuous for every $s>-1 / 2$.

(2) By using Rempel-Schulze [21, p. 102, Proposition 6 and p. 151, Proposition 10], we find from formula $(9.8)$ that the adjoint $\left(\gamma_{0} \mathcal{G}_{N}\right)^{*}$ is a potential operator of order -2 with the principal symbol which is homotopic to the following:

$$
-\frac{1}{\left\langle\xi^{\prime}\right\rangle} \frac{1}{\left\langle\xi^{\prime}\right\rangle+i \nu}
$$

Hence, it follows from an application of $[21$, p. 156, Theorem 4] that the adjoint

$$
\left(\gamma_{0} \mathcal{G}_{N}\right)^{*}: H^{s+1 / 2}(\Gamma) \longrightarrow H^{s+2}(\Omega)
$$

is continuous for every $s \in \mathbf{R}$. 
(3) By using Rempel-Schulze [21, p. 102, Proposition 6 and p. 151, Proposition $10]$, we find from formula (8.8) that the adjoint $\left(\mathcal{P}_{1}\right)^{*}$ is a trace operator of order -1 and type 0 with the principal symbol which is homotopic to the following:

$$
-\frac{1}{\left\langle\xi^{\prime}\right\rangle-i \tau} \text {. }
$$

Hence, it follows from an application of [21, p. 158, Theorem 5] that the adjoint

$$
\left(\mathcal{P}_{1}\right)^{*}: H^{s}(\Omega) \longrightarrow H^{s+1 / 2}(\Gamma)
$$

is continuous for every $s>-1 / 2$.

(4) Finally, we find from assertions (9.9) and (9.10) that the adjoint $\left(T_{1}^{-1} b\left(x^{\prime}\right)\right)^{*}$ is a pseudo-differential operator in $L_{1,1 / 2}^{0}(\Gamma)$ with the principal part which is homotopic to the following:

$$
b\left(x^{\prime}\right) \overline{s_{0}\left(x^{\prime}, \xi^{\prime}\right)}=\frac{b\left(x^{\prime}\right)}{\overline{t_{0}\left(x^{\prime}, \xi^{\prime}\right)}},
$$

and further that the adjoint operator

$$
\left(\mathcal{T}_{1}{ }^{-1} b\left(x^{\prime}\right)\right)^{*}: H^{s+1 / 2}(\Gamma) \longrightarrow H^{s+1 / 2}(\Gamma)
$$

is continuous for every $s \in \mathbf{R}$.

By combining assertions (10.3). (10.4), (10.6) and (10.5), we obtain that the adjoint operator

$$
\mathcal{G}^{*}=\left(\mathcal{G}_{N}\right)^{*}-\left(\gamma_{0} \mathcal{G}_{N}\right)^{*}\left(\mathcal{T}_{1}^{-1} b\left(x^{\prime}\right)\right)^{*}\left(\mathcal{P}_{1}\right)^{*},
$$

is continuous for every $s \geq 0$.

The proof of Theorem 10.1 is complete.

\subsection{End of Proof of Theorem 2.2}

By Corollary 9.1 and Theorem 10.1, we find from Remark 4.1 that the closed realization $\mathcal{A}:=\mathfrak{A}-\lambda_{1} I$ satisfies condition (B) in Theorem 4.1.

Therefore, the desired assertions (i), (iii), (iv) and (v) of Theorem 2.2 follow from an application of Theorem 4.1 with $\mathcal{A}:=\mathfrak{A}-\lambda_{1} I$.

Now the proof of Theorem 2.2 is complete.

\section{Concluding remark}

Finally, we refer to the works of Grubb-Kokholm [10] and Krietenstein-Schrohe [15] for an extensive further development of the present paper in the framework of Besov and Sobolev spaces of $L^{p}$ type (see also Grubb [9] for $L^{2}$-based Sobolev spaces).

Acknowledgements The author is grateful to the referee for many valuable comments and suggestions which improved the presentation of this paper, both mathematically and stylistically, and for informing him of some additional references. 


\section{References}

1. Adams, R.A., Fournier, J.J.F.: Sobolev spaces, second edition. Pure and Applied Mathematics, Elsevier/Academic Press, Amsterdam (2003)

2. Agmon, S.: Lectures on elliptic boundary value problems. Van Nostrand, Princeton, New Jersey (1965)

3. Bourdaud, G.: $L^{p}$-estimates for certain non-regular pseudo-differential operators. Comm. Partial Differential Equations 7, 1023-1033 (1982)

4. Boutet de Monvel, L.: Boundary problems for pseudo-differential operators. Acta Math. 126, 11-51 (1971)

5. Chazarain, J., Piriou, A.: Introduction à la théorie des équations aux dérivées partielles linéaires. Gauthier-Villars, Paris (1981)

6. Fujiwara, D., Uchiyama, K.: On some dissipative boundary value problems for the Laplacian. J. Math. Soc. Japan 23, 625-635 (1971)

7. Gilbarg, D., Trudinger, N.S.: Elliptic partial differential equations of second order, reprint of the 1998 edition. Classics in Mathematics, Springer-Verlag, New York Berlin Heidelberg Tokyo (2001)

8. Greiner, P.: An asymptotic expansion for the heat equation. Arch. Rational Mech. Anal. 41, 163-218 (1971)

9. Grubb, G.: Functional calculus of pseudodifferential boundary problems. Progress in Mathematics, Vol. 65. Birkhäuser Boston, Inc., Boston, Massachusetts (1986)

10. Grubb, G., Kokholm, N.J.: A global calculus of parameter-dependent pseudodifferential boundary problems in $L_{p}$ Sobolev spaces. Acta Math. 171, 165-229 (1993)

11. Hörmander, L.: Pseudo-differential operators and hypoelliptic equations. In: Proc. Sym. Pure Math., X, Singular integrals, A.P. Calderón (ed.), 138-183. American Mathematical Society, Providence, Rhode Island (1967)

12. Hörmander, L.: The analysis of linear partial differential operators III, Pseudo-differential operators, reprint of the 1994 edition, Classics in Mathematics, Springer-Verlag, Berlin Heidelberg New York Tokyo (2007)

13. Iwasaki, C.: The asymptotic expansion of the fundamental solution for parabolic initialboundary value problems and its application. Osaka J. Math. 31, 663-728 (1994)

14. Kannai, Y.: Hypoellipticity of certain degenerate elliptic boundary value problems. Trans. Amer. Math. Soc. 217, 311-328 (1976)

15. Krietenstein, T., Schrohe, E.: Bounded $H^{\infty}$-calculus for a degenerate elliptic boundary value problem.

16. Kumano-go, H.: Pseudodifferential operators. MIT Press, Cambridge, Massachusetts (1981)

17. Lions, J.-L., Magenes. E.: Problèmes aux limites non-homogènes et applications 1, 2. Dunod, Paris (1968)

18. Mizohata, S.: The theory of partial differential equations. Cambridge University Press, London, New York (1973)

19. Munkres, J.R.: Elementary differential topology. Annals of Mathematics Studies, No. 54. Princeton University Press, Princeton, New Jersey (1966)

20. Protter, M.H., Weinberger, H.F.: Maximum principles in differential equations, corrected second printing. Springer-Verlag, New York (1984)

21. Rempel, S., Schulze, B.-W.: Index theory of elliptic boundary problems. Akademie-Verlag, Berlin (1982)

22. Schrohe, E.: A short introduction to Boutet de Monvel's calculus. In: Approaches to Singular Analysis, J. Gil, D. Grieser and M. Lesch (eds), 85-116, Oper. Theory Adv. Appl., 125, Birkhäuser, Basel (2001)

23. Seeley, R.T.: Singular integrals and boundary value problems. Amer. J. Math. 88, 781-809 (1966)

24. Seeley, R.T.: Topics in pseudo-differential operators. In: Pseudo-differential operators (C.I.M.E., Stresa, 1968), L. Nirenberg (ed.), 167-305. Edizioni Cremonese, Roma (1969). Reprint of the first edition, Springer-Verlag, Berlin Heidelberg (2010)

25. Stein, E.M.: Singular integrals and differentiability properties of functions. Princeton Mathematical Series, no. 30, Princeton University Press, Princeton, New Jersey (1970)

26. Taira, K.: On some degenerate oblique derivative problems. J. Fac. Sci. Univ. Tokyo, Sec. IA 23, 259-287 (1976)

27. Taira, K.: Semigroups, boundary value problems and Markov processes, second edition. Springer Monographs in Mathematics. Springer-Verlag, Berlin Heidelberg New York (2014) 
28. Taira, K.: Analytic semigroups and semilinear initial-boundary value problems, second edition. London Mathematical Society Lecture Note Series, vol. 434. Cambridge University Press, Cambridge (2016)

29. Taira, K.: Spectral analysis of the subelliptic oblique derivative problem. Ark. Mat. 55, 243-270 (2017)

30. Taylor, M.E.: Pseudodifferential operators. Princeton Mathematical Series, Vol. 34, Princeton University Press, Princeton, New Jersey (1981)

31. Wloka, J.: Partial differential equations. Cambridge University Press, Cambridge (1987) 